\title{
Reliable Langmuir Blodgett colloidal masks for large area nanostructure realization
}

\author{
Madasamy Thangamuthu ${ }^{a, b *}$, Christian Santschi ${ }^{a}$, and Olivier J.F. Martin ${ }^{a *}$

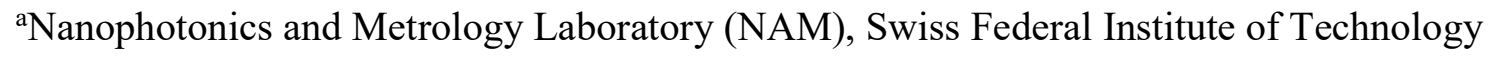 \\ Lausanne (EPFL), CH-1015 Lausanne, Switzerland.

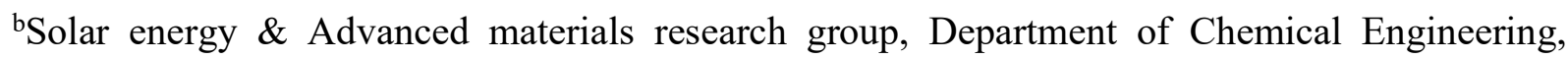 \\ University College London (UCL), WC1E 7JE London, United Kingdom. \\ $\underline{\text { *m.thangamuthu@ucl.ac.uk, }}$ *olivier.martin@epfl.ch
}

\begin{abstract}
The fabrication of highly ordered nanostructures over large areas is key for many technologies and colloidal lithography using the Langmuir Blodgett technique appears a simple and straightforward way of reaching that goal. While this technique has been widely reported in the literature, its straightforward implementation to obtain well-ordered nanostructures over very large areas is far from obvious, since many key technical subtleties are rarely documented. Here, we describe an easily and highly reproducible recipe and detail aspects such as beads preparation, composition of the subphase, beads transfer method, influence of the spreading agent and the barrier compression rate, as well as monolayer transfer to the substrate. A drastic improvement in the polystyrene self-assembly at the air-water interface is observed after removing the common salt and surfactant molecules from commercial polystyrene beads suspensions. Similarly, an electrolyte free water subphase enhances the hexagonal arrangement of the beads and the longrange order. The beads sinking into the bulk of the water is reduced by dispensing the beads using a glass slide and the polystyrene suspension prepared using water and ethanol at 1:1 mitigates repulsive and attractive forces, leading to excellent hexagonal close packed arrangement. By following the recipe shown here, the reader should easily fabricate lattice-like colloidal masks for producing nanostructures over larger areas.
\end{abstract}

\section{Keywords}

Colloidal lithography; Langmuir-Blodgett; 2D assembly; Polystyrene beads; Water-air interface; Large area plasmonic nanostructures. 


\section{Introduction}

Fabrication of highly ordered nanostructures over large areas is key for many applications including nanoelectronics, optics, plasmonics, photocatalysis, photovoltaics, and biology [1-4]. These applications require the fabrication of nanostructures over very large areas, up to $\mathrm{cm}^{2}$ and beyond. Unfortunately, the commonly used lithographic techniques, viz. electron beam $[5,6]$, ion beam [7,8], and photolithography [9] can fabricate well-defined nanostructures only over limited areas, in the order of a few hundred $\mu \mathrm{m}^{2}$. Furthermore, these methods are very expensive and time consuming for nanostructure mass production. To overcome these shortcomings, colloidal lithography, also known as nanosphere lithography (NSL), is an extremely appealing technique for large scale fast pattering of periodic nanostructures. This technique was first used by Fischer and Zingsheim to demonstrate contact optical lithography with sub-micron resolution [10]. It has then been used to fabricate microporous materials [11] and periodic apertures in silicon films [12]. Since then, its popularity has grown immensely and the technique has been reviewed by several authors [13-17]. NSL is very popular in plasmonics, where it can be used to produce wellcontrolled metallic nanostructures over large areas [18-20]. Further refinements that rely on the third dimension of NSL and non-normal evaporation techniques have enabled a wealth of nanostructures with interesting optical properties [21-25]. In a typical NSL process, a hexagonal close packed (hcp) 2D colloidal mask is formed at an air-water interface and then transferred to the substrate, followed by metal evaporation/sputtering and lift-off process, leading to basic triangular nanostructures [26]. More sophisticated shapes such as nanodots, nanorings, nanospheres, nanopillars, and crescent shaped particles can also be obtained using NSL [27-30]. To obtain such a nanostructure in an ordered manner over large scale, fabrication of high quality hep mask is crucial [25].

Several methods have been shown to prepare closely packed as well as non-closely packed nanosphere masks [16,31]. Spin coating is commonly used because of its simplicity [32]. However, the formation of quasi-double layer is unavoidable and also the ordered domain size is limited, resulting in poor homogeneity [33]. To address these issues, an interfacial self-ordering approach has been used in which highly ordered uniform masks can be prepared by manual addition of nanospheres at an air-water interface and solidify by adding a suitable surfactant before transferring to the substrate [34]. Though this has issues like beads sinking into the bulk of the water, while the presence of surfactant molecules can affect the optical properties of the 
nanostructures. A high quality hep mask without adding solidifying agent/surfactant can be obtained using the Langmuir Blodgett (LB) technique [35]. This approach is well known for producing colloidal masks with excellent homogeneity over large areas. It consists of dispensing the particle suspension onto the water surface with barriers in open position, allowing the particles to equilibrate and self-assemble to form a monolayer, followed by compression before transferring to the substrate [36].

Although several reports demonstrate the LB technique for hcp mask preparation, the given procedures seem not reproducible due to the lack of information on specific parameters involved in this approach [37-42]. Some controversy can also be found in the literature, for instance, Williams et al. demonstrated that even a small concentration of salt in water would result in aggregation of polystyrene (PS) particles [43]. In contrast, Robinson et al. claimed no aggregation of the particles although the water had high salt concentration [44]. Molecular dynamics simulations and modeling have also been used to study the inter-particles interaction and the role of polydispersity $[38,45]$. Another study shows that higher hydrophobicity can increase the stability of the monolayer thanks to the electrostatic and steric repulsion between the particles [46]. The role of spreading solvent, the effect of $\mathrm{pH}$, and subphase temperature have also been studied [47-49]. The colloidal mask transfer to the substrate has also been shown by means of i) rapid immersion of hydrophilic substrate and then slowly withdrawn using dip coater, ii) lowering the subphase, and iii) fully evaporating the water until particles deposit on the substrate [50-52]. Most of these works use silica particles and some are only simulations, not giving detailed information about all parameters involved in the experiments.

Our frustration in trying to reproduce some published recipes for the realization NSL masks using the LB approach and the ensuing discovery of key experimental parameters that are rarely documented, has motivated this study, where we optimize and describe in detail several facets of the LB technique for the realization of NSL masks, including i) modifying the surface chemistry of the beads, ii) the preparation of the water subphase, iii) the beads spreading methods, iv) the spreading agent composition, v) the barrier compression, and the hcp mask transfer to the substrate. We optimized all these conditions for commercially available negatively charged PS beads and achieved high quality 2D masks with high packing density and very small defects. Finally, highly ordered aluminium nanostructures were fabricated over a $2.5 \mathrm{~cm}^{2}$ glass substrate using these masks. 


\section{Experimental details}

\subsection{Materials and reagents}

Ammonia, hydrogen peroxide, isopropyl alcohol, ethanol and acetone were purchased from Sigma Aldrich (Buchs, Switzerland) and used without further purification. A 5\% aqueous suspension of polystyrene (PS) beads with diameter $340 \mathrm{~nm}$, non-functionalized spheres containing a slight anionic charge from sulfate ester, was purchased from Microparticles GmbH, Germany. Deionised (DI) water was used as subphase for the LB trough and other reagents preparation. Ethanol was used as spreading agent. An ultrasonic homogeniser UP50H from Hielscher was used to redisperse the decanted PS beads. The LB setup (Kibron micro trough G4) used to fabricate hcp PS 2D colloidal masks consists of dual compression barriers and a LayerX dip coater.

\subsection{Beads washing}

Commercial PS beads were washed using rinse method to remove the water-soluble surfactant and salts present in the suspension. Briefly, $200 \mu \mathrm{L}$ of the suspension was pipetted out into the $1.5 \mathrm{~mL}$ Eppendorf tube and mixed with $800 \mu \mathrm{L}$ of spreading agent ethanol. This mixture was centrifuged at $6000 \mathrm{rpm}$ for $15 \mathrm{~min}$ at $4{ }^{\circ} \mathrm{C}$ followed by decanting the supernatant liquid. To re-disperse the beads, $1000 \mu \mathrm{L}$ of fresh ethanol was added followed by 2 min ultrasonic pulses. This process was repeated three times. Then, the final beads were re-dispersed in water-ethanol solution at 1:1 ratio. Figure 1 explains the schematic of the beads washing process in detail.
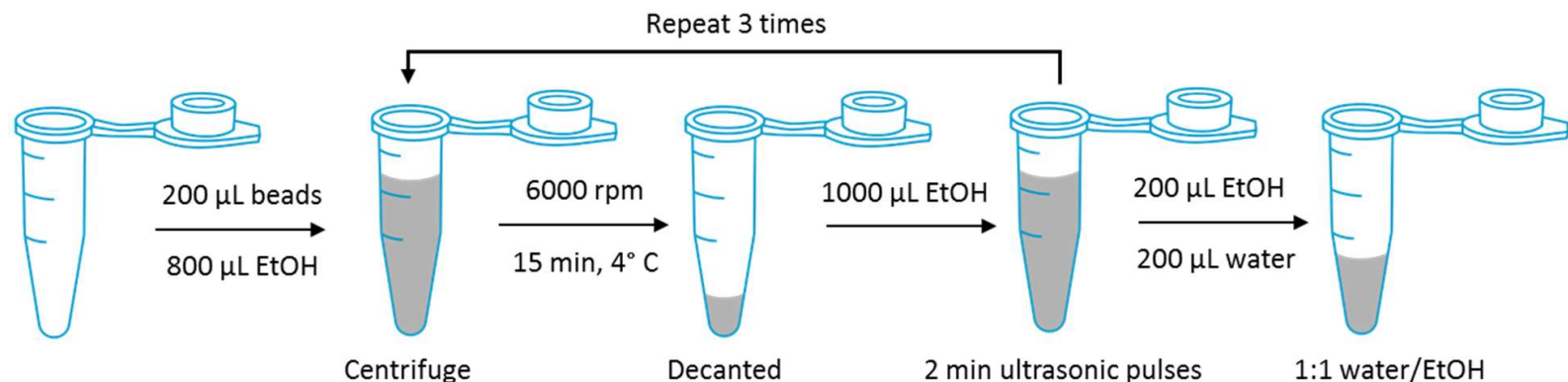

Fig. 1. Schematic representation of the steps involved in PS beads washing process.

\subsection{Substrate pre-treatment}

Substrate pre-treatment is essential to remove residual organics on the surface and render the surface hydrophilic. Briefly, float glass wafers were pre-treated in a mixture of $20 \mathrm{~mL} \mathrm{NH} \mathrm{NH}_{4} \mathrm{OH}$ 
(25\%): $10 \mathrm{~mL} \mathrm{H} \mathrm{H}_{2} \mathrm{O}: 5 \mathrm{~mL} \mathrm{H} \mathrm{H}_{2}(30 \%)$ solution at $75^{\circ} \mathrm{C}$ for $30 \mathrm{~min}$. The pre-treated wafers were diced into $2.5 \times 2.5 \mathrm{~cm}^{2}$ pieces.

\subsection{LB trough initialization}

Before filling the LB trough with water, it was cleaned using deionised water followed by ethanol and dried using nitrogen gas. Then, the DI water was filled until slightly overfilling to obtain a better sealing between the hydrophobic barriers and the water subphase. The purity of the air-water interface was monitored by measuring the surface pressure $(\pi)$ using the trough sensor probe. A laboratory aspirator (Benchmark Scientific V0020) was used to remove the impurities at the interface until the surface pressure decreased to $0.5-1 \mathrm{mN} \mathrm{m}^{-1}$.

\subsection{Realization of $2 D$ colloidal mask}

After LB trough initialization, $200 \mu \mathrm{L}$ of washed PS beads suspension was taken in a micropipette and dispensed at the air-water interface using a glass slide positioned on either side of the barrier at an angle of $\sim 45^{\circ}$ with respect to the water surface. The beads suspension was spread out at the interface as drops with time interval of $30 \mathrm{~s}$ (it typically takes $20 \mathrm{~min}$. to fill the trough). Subsequently, the PS spheres roll down to the water surface and self-assemble. The beads were then allowed to equilibrate for $20 \mathrm{~min}$. Due to the electrostatic repulsion between the adjacent beads and the attractive capillary force (deformation of the liquid meniscus by electrostatic stress) [53], hexagonally arranged smaller domains were obtained. Then, a compression isotherm was carried out by moving both barriers towards the middle of the trough resulting in the coalescence of the smaller hcp domains into a larger one. The compressing movement was stopped just before reaching the collapsing point (surface pressure above $20 \mathrm{mN} \mathrm{m}^{-1}$ ) of the hcp monolayer. After a short breathing time, the hcp monolayer was transferred to the glass substrate using the LayerX dip coater and dried in a nitrogen chamber. The schematic illustration of the PS colloidal mask realization is shown in Fig. 2. 

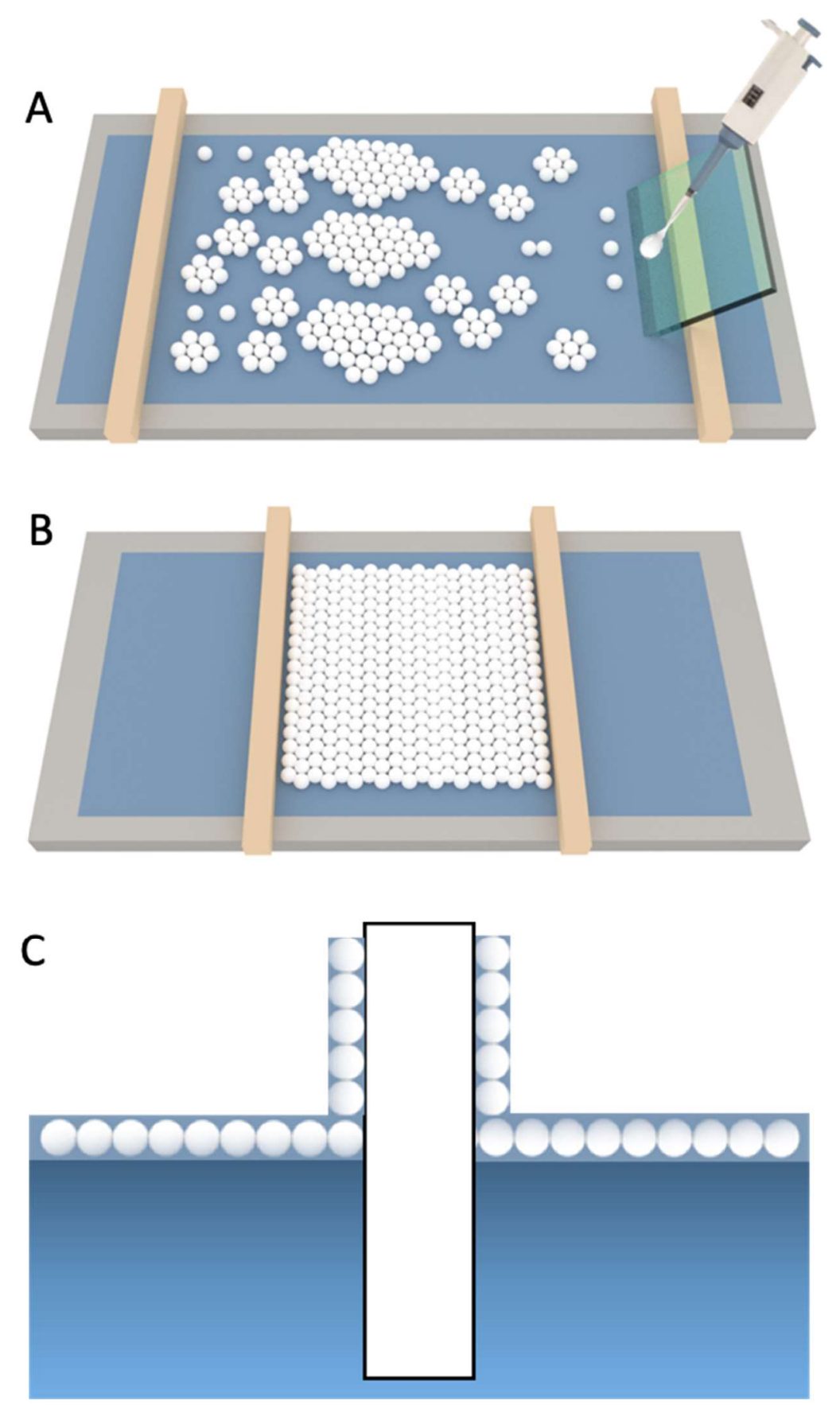

Fig. 2. Schematic illustration of the LB colloidal mask preparation processes. (A) PS beads spreading at the air/water interface forms a self-assembled hexagonally close packed (hcp) arrangement. (B) The barriers compression arranges smaller hcp domains into a larger homogeneous domain. (C) The hcp colloidal mask is transferred onto the substrate.

\subsection{Aluminium nanostructures realization}

After 2 days drying, an Al thin film (40 nm) was deposited on the hcp PS mask using electron beam evaporation with a base pressure between $1.33 \times 10^{-4}$ and $10^{-5}$ Pascal (Leybold Optics 
LAB600H). Highly ordered aluminium nanotriangles (AlNTs) were then obtained by lift-off the mask in toluene for 10 min under ultrasonication.

\subsection{Characterization}

The self-assembly and close packing arrangement (crystallinity) of the beads on the substrates at different working conditions were examined by scanning electron microscope (SEM) images. The SEM images were recorded using a Zeiss Merlin field emission scanning electron microscope (Carl Zeiss Jena $\mathrm{GmbH}$ ) with $2 \mathrm{keV}$ electron energy. The Fourier transform images and Voronoi diagrams were obtained using the statistical image analysis software ImageJ. The optical properties (extinction) of the aluminium nanostructures were studied using a UV-Vis-NIR spectrometer (Shimadzu, UV-2600).

\section{Results and discussion}

\subsection{Effect of beads washing}

The surface chemistry is a crucial parameter to control the floating properties of the beads at the air-water interface. The washing process described in section 2.2 alters the surface chemistry of the commercial PS beads suspension. Its influence on the PS monolayer formation was studied and the results are shown in Fig. 3. When dispensing the beads without washing, we noticed that most of the beads sank into the water, which became whitish. Furthermore, the SEM image (Fig. $3 \mathrm{~A}$ ) of the substrate prepared by this condition shows no beads on it suggesting that no monolayer is formed. This is due to the presence of water-soluble surfactants and salts in the PS suspension, leading to the immersion of the beads into the water. Fig. 3B shows the SEM image of the PS monolayer obtained using the beads after one single wash. It can be clearly seen that certain beads were self-assembled although the packing is still very poor. We also noticed visually that the water still becomes somewhat whitish in that case. Hence, we washed the beads again three times and observed excellent floating at the interface and outstanding PS self-assembly (Fig. 3C), thanks to the hydrophobic nature of the beads and the negligible amount of water-soluble contents in the suspension. Although PS beads have a slightly higher mass density than water, beads remain at the surface due to the low surface tension as a result of the washing cycles. A more in-depth analysis of the beads arrangement transferred to the substrate was performed by processing the SEM images, in particular, the degree of ordering was examined by analysing the Fast Fourier Transform (FFT) images as shown in the insets of Fig. 3A, B, and C. The first-order hexagonal 
Bragg peaks observed for the mask prepared using properly washed beads (inset of Fig. 3C) indicate a uniform beads lattice demonstrating an excellent degree of order. Furthermore, the structural homogeneity of the PS arrangement was evaluated by Voronoi-diagram as shown in Fig. 3D, E, and F. The perfect honeycomb pattern with equilateral hexagons (Fig. 3F) was observed for the monolayer obtained by the washed beads revealing the hexagonal pattern of identical PS beads (each bead surrounded by six beads).
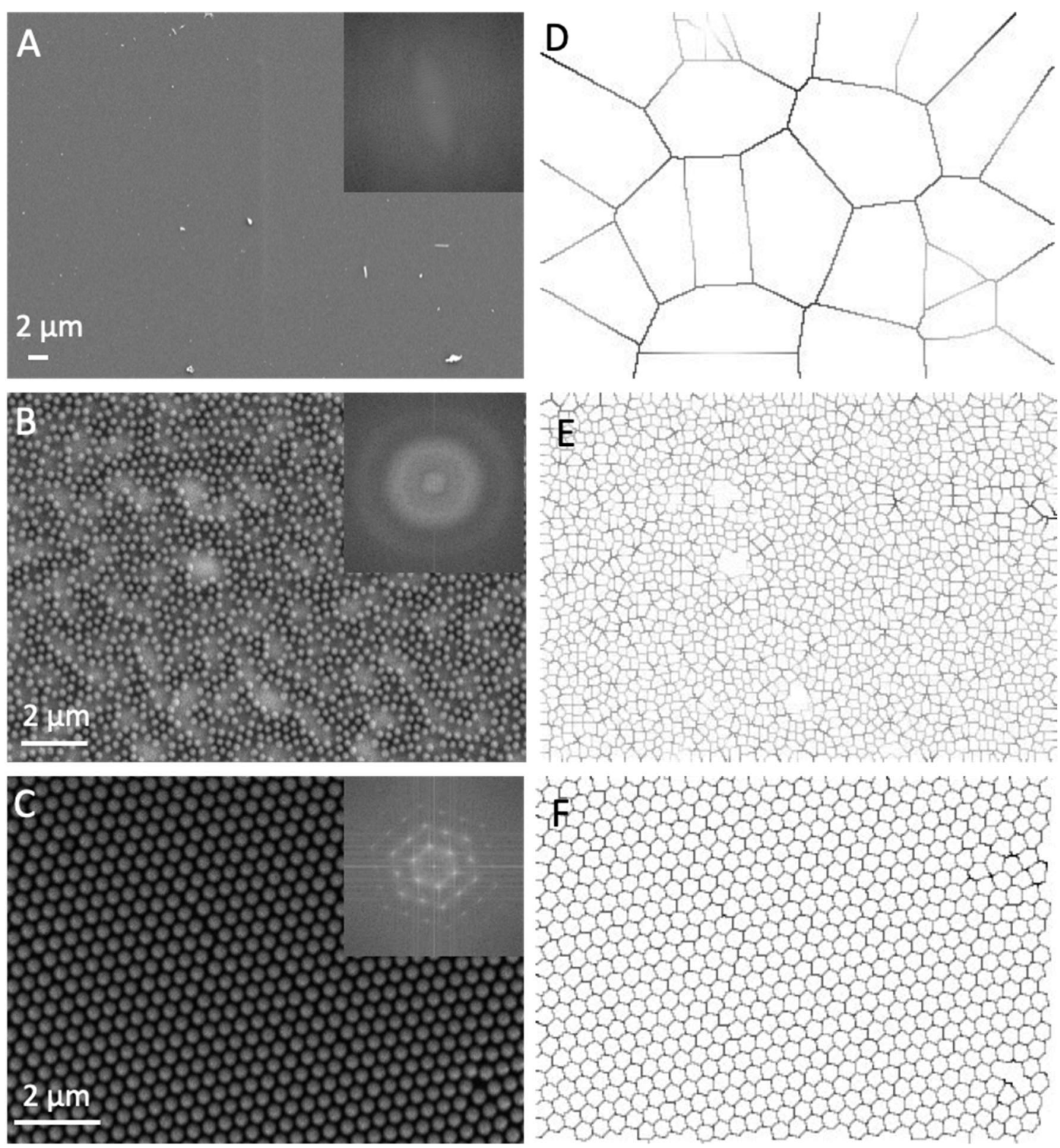

Fig. 3. SEM and corresponding FFT images (insets) showing the influence of the bead washing process on the PS monolayer formation (images are taken after transferring the monolayer to the substrate). (A) No wash; (B) after one wash and (C) four washes cycles. (D), (E), and (F) show the Voronoi-diagram of the (A), (B), and, (C) SEM images, respectively. 
The lower order observed for a monolayer prepared using unwashed beads (Fig. 3D) or after a single wash (Fig. 3E) denote the disappearance of the hexagonal order. Those results suggest that the water-soluble contents in suspension must be removed to obtain high quality PS colloidal masks.

\subsection{Does the subphase water need electrolyte?}

As discussed in the introduction, there are different opinions regarding the choice of the water subphase. It is known that the electrostatic repulsion between adjacent particles and the attractive capillary force (due to the deformation of the liquid meniscus by electrostatic stress) self-assemble the beads in an hcp arrangement over long range order [53]. To better understand the role of electrolyte in the water subphase, we first dispensed unwashed beads at the air-water interface for different sodium chloride $(\mathrm{NaCl})$ concentrations. In the absence of salt, unwashed beads were totally immersed into the water due to the presence of water-soluble surfactant and salt in the suspension and the SEM image of the corresponding substrate shows no beads (data not shown). However, a significant number of floating beads were observed in the presence of NaCl. Fig. 4AD show the SEM images of the PS floating layer prepared using unwashed beads in the presence of $6 \%, 2 \%, 1.5 \%$ and $1 \%$ of the salt, respectively. It is obvious that a higher salt concentration (Fig. 4A) lead to the formation of beads cluster and poor monolayer packing that can be explained by the fact that in the presence of higher electrolyte strength in the subphase, more charge screening of the beads (electrostatic repulsion between the particles is screened) occurs leading to the beads accumulation by attractive van der Waals forces [54]. The hydration force (a strong short-range repulsive force) due to the electrolyte is also believed to cause aggregation. Reducing the salt concentration to $1 \%$ (Fig. 4D) shows little aggregation (mostly doublet and triplet), suggesting that the surface charge density of the electrolyte is still higher than the native beads, which have only little sulfate anion (see section 2.1 for more information). After a further reduction of the $\mathrm{NaCl}$ concentration, below $1 \%$, the beads partly immerse into the water demonstrate the importance of the washing step to control the floating properties of the beads. Hence, we compared the formation of PS monolayers using washed beads in the presence and absence of $1 \%$ dissolved $\mathrm{NaCl}$. In the presence of the salt, although some aggregates were found (Fig. 4E), significant improvement of the order of the monolayer arrangement was observed; whereas in its absence a very nice hep packing was obtained (Fig. 4F). This indicates that washing the beads efficiently 
removes the water-soluble contents in the suspension and the remaining, negative surface charges prevent agglomeration by electrostatic repulsion and capillary forces, pull the beads together by evaporation of the solvent in interstices to form hcp pattern. Therefore, commercial beads with no special functionalization, after washing, do not require the addition of salt in the subphase to achieve excellent PS beads packing.
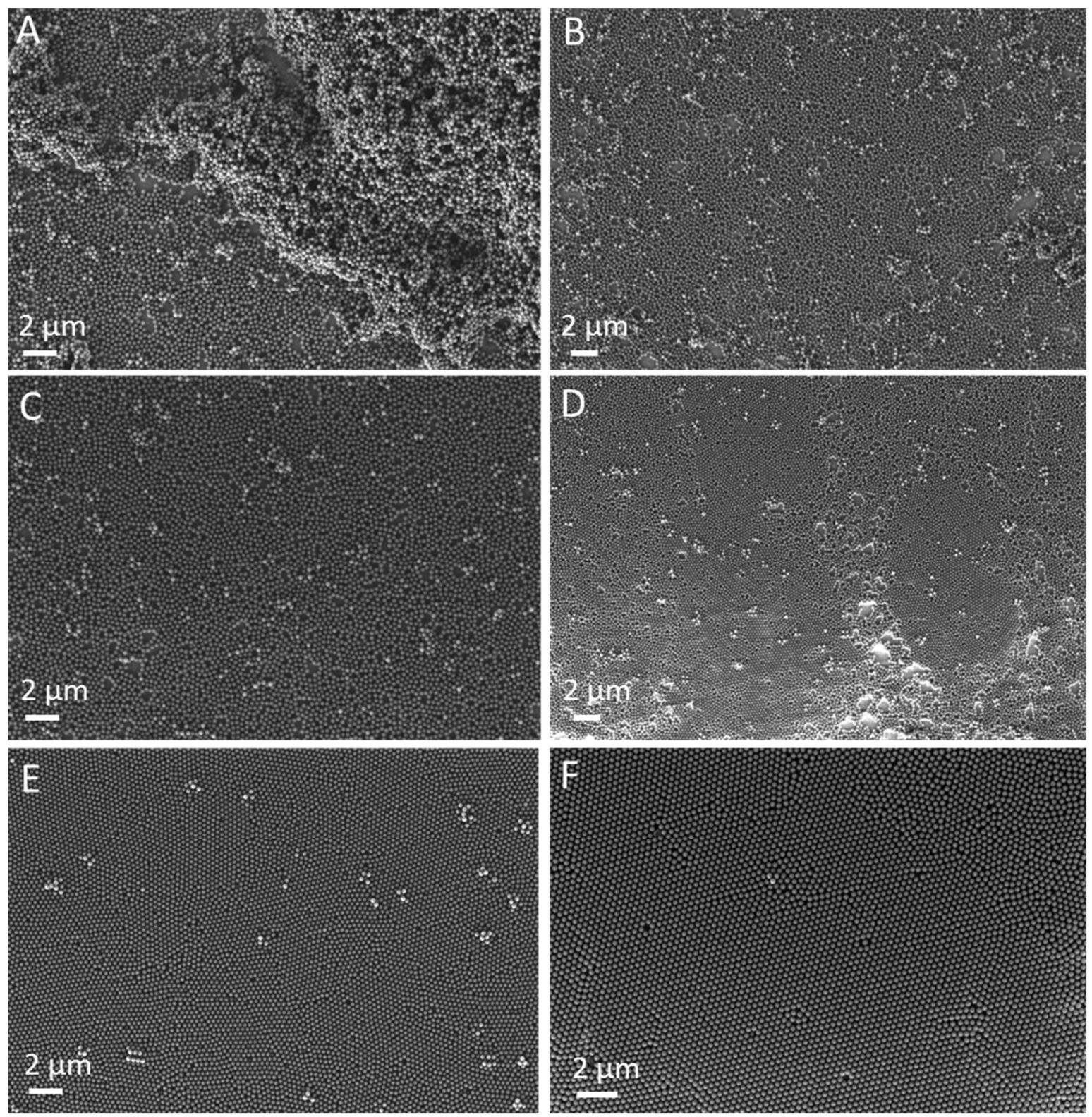

Fig. 4. SEM images showing the influence of salt concentration in water on the PS monolayer formation at the air-water interface. (A)-(D), unwashed beads were spread on water subphases containing different $\mathrm{NaCl}$ concentrations: (A) $6 \%$, (B) $2 \%$, (C) $1.5 \%$ and (D) $1 \%$. (E) and (F) the washed beads were spread onto the water containing: (E) $1 \% \mathrm{NaCl}$ and (F) no salt. 


\subsection{Method of dispensing}

The method of dispensing influences the PS monolayer formation since interferences at the airliquid interface affect the quantity of beads immersing into the water although they are washed. In the present study, we have used two dispensing strategies i) dropping the beads directly onto the surface, and ii) using a glass slide tilted at $45^{\circ}$ as shown in Fig. $5 \mathrm{~A}$, and B, respectively.
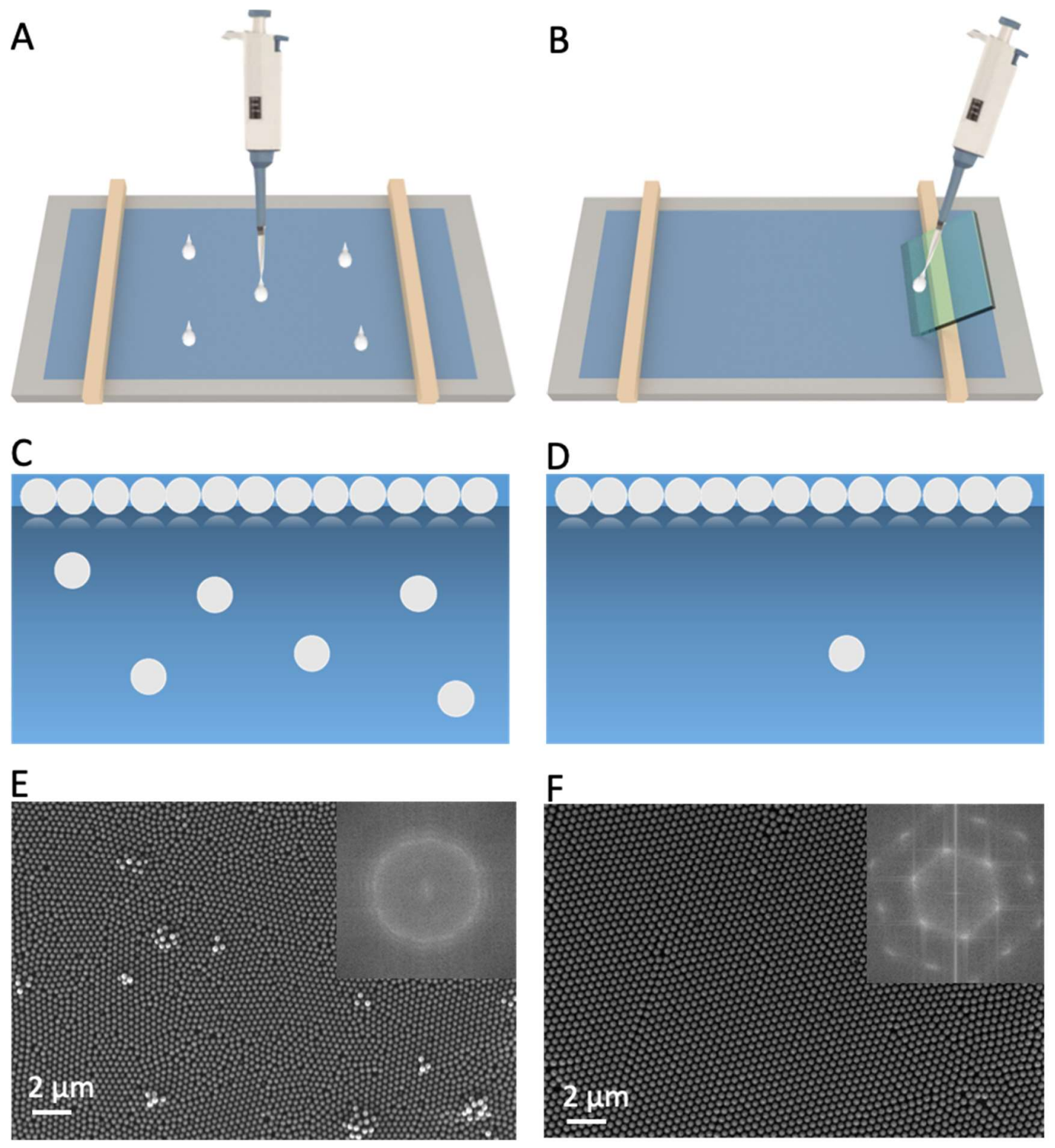

Fig. 5. Optimization of the beads spreading method: (A), (C) and (E) show the schematic illustration of the beads dispensed directly at the air-water interface, leading to the beads sinking into the water, and the corresponding SEM image of the resulting monolayer. (B), (D), and (F) show the schematic illustration of beads dispensed using a glass slide angled at $45^{\circ}$ with respect to the air-water interface and the corresponding SEM image of the resulting monolayer. Insets of Fig. 5E and F show the FFT images of the corresponding SEM images. 
While dispensing the washed beads directly as small drops at $30 \mathrm{~s}$ time intervals, the turbulences push the beads into the water [55], as schematically represented in Fig. 5C. Using this method, the beads can agglomerate into small domains as indicated in the SEM image (Fig. 5E). To overcome this effect, we used a glass slide angled at $45^{\circ}$ to the water surface in which part of the glass slide was immersed into the water and the beads were dispensed over the glass slide slightly above the water surface, which significantly reduced the beads immersion as shown schematically in Fig. 5D. The SEM image obtained using the glass slide assisted dispensing method produces a nice hep monolayer (Fig. 5F), which agrees well with the literature [56]. Furthermore, the degree of order observed using FFT image for the glass slide-assisted dispensing method (inset of Fig. 5F) shows well-defined Bragg peaks, compared to the FFT image for the direct dispensing method (inset of Fig. 5E), evidencing the higher order. Hence, we recommend the method sketched in Fig. 5B as optimized dispensing method.

\subsection{Effect of spreading agent}

To understand the role of the spreading agent on the particle's arrangement at the air-water interface, we prepared a PS suspension in the presence or the absence of ethanol, and in a water/ethanol mixture at 1:1 ratio.

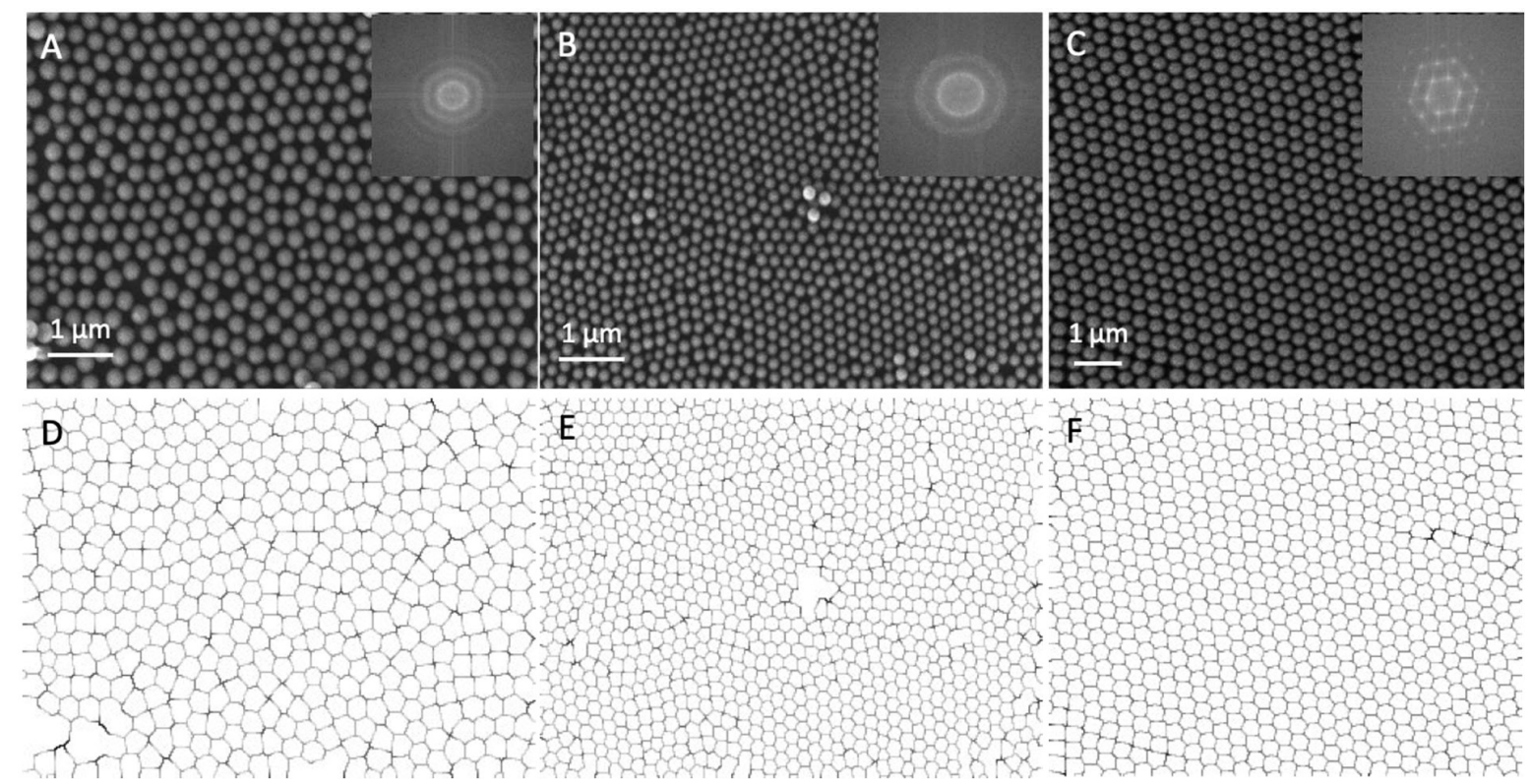

Fig. 6. SEM and corresponding FFT images (insets) of the PS monolayer obtained using the suspension prepared in: (A) water, (B) ethanol and (C) ethanol/water at 1:1 ratio. D, E, and F show the Voronoi diagram derived from the A, B, and C SEM images respectively. 
The PS monolayer obtained in the absence of ethanol exhibits non-closely packed PS arrangements, Fig. 6A. The FFT image (inset in Fig. 6A) and Voronoi diagram (Fig. 6D) derived from the SEM image [57] reveal the low ordering and poor hexagonal arrangements respectively. This is because in the absence of ethanol, the repulsive force between the beads is dominating, thus preventing the formation of a close packed arrangement. In the case of suspension prepared only in ethanol, attractive forces due to the capillary action upon evaporation play a dominant role and particles form short-range hcp arrangements as shown in Fig. 6B. It is also evident from the short range honeycomb domains observed by Voronoi diagram, Fig. 6E. Mitigating attractive and repulsive forces is required to obtain both short and long-range ordering; this is achieved by using the beads prepared in water/ethanol solution at 1:1 ratio [58]. Fig. $6 \mathrm{C}$ shows excellent arrangement of the hcp monolayer, with clear first-order Bragg peaks in FFT image (inset of Fig. 6C) and Fig. $6 \mathrm{~F}$ reveals excellent hexagonal pattern for the beads arrangement, suggesting that the beads suspension must be prepared in spreading agent and water in equal proportions.

\subsection{Barrier compression and beads transfer to the substrate}

One of the important advantages of the LB approach is to obtain a 2D colloidal crystal mask with long-range order by compressing the smaller domains. This avoids the usage of solidifying agent or surfactant to form a lattice-like arrangement. The barrier compression phase diagram (Surface pressure vs. Area) exhibits typically three regions. In region 1, the surface pressure shows a slow rise corresponding to large areas; then in region 2, the surface pressure rise is steeper since it is due to the inter particle electrostatic repulsion upon compression; in region 3, the abrupt surface pressure changes due to the collapse of the monolayer. To achieve a long-range hcp monolayer, it is important to optimize the compression speed without damaging the monolayer. Before dispensing the beads at the air-water interface the surface pressure was kept below $1 \mathrm{mN} \mathrm{m}^{-1}$ (Fig. 7A). Fig. 7B exhibits a compression isotherms observed for PS monolayer at different barrier speeds from $30 \mathrm{~mm} \mathrm{~min}^{-1}$ to $5 \mathrm{~mm} \mathrm{~min}^{-1}$. At higher speed, the surface pressures were not increased uniformly due to the monolayer collapse. At slower compression speeds, a slow increase of the surface pressure was observed. The exponential rise of the surface pressure below an area of 7000 $\mathrm{mm}^{2}$ confirms that all smaller domains are well connected over long-range, without damaging the monolayer. At a compression speed of $10 \mathrm{~mm} \mathrm{~min}^{-1}$, a maximum surface pressure of $20 \mathrm{mN} \mathrm{m}^{-1}$ was obtained. After further compression the hep monolayer collapsed. We can conclude that 10 $\mathrm{mm} \mathrm{min}-1$ is the optimized speed for barrier compression to obtain larger domains. 

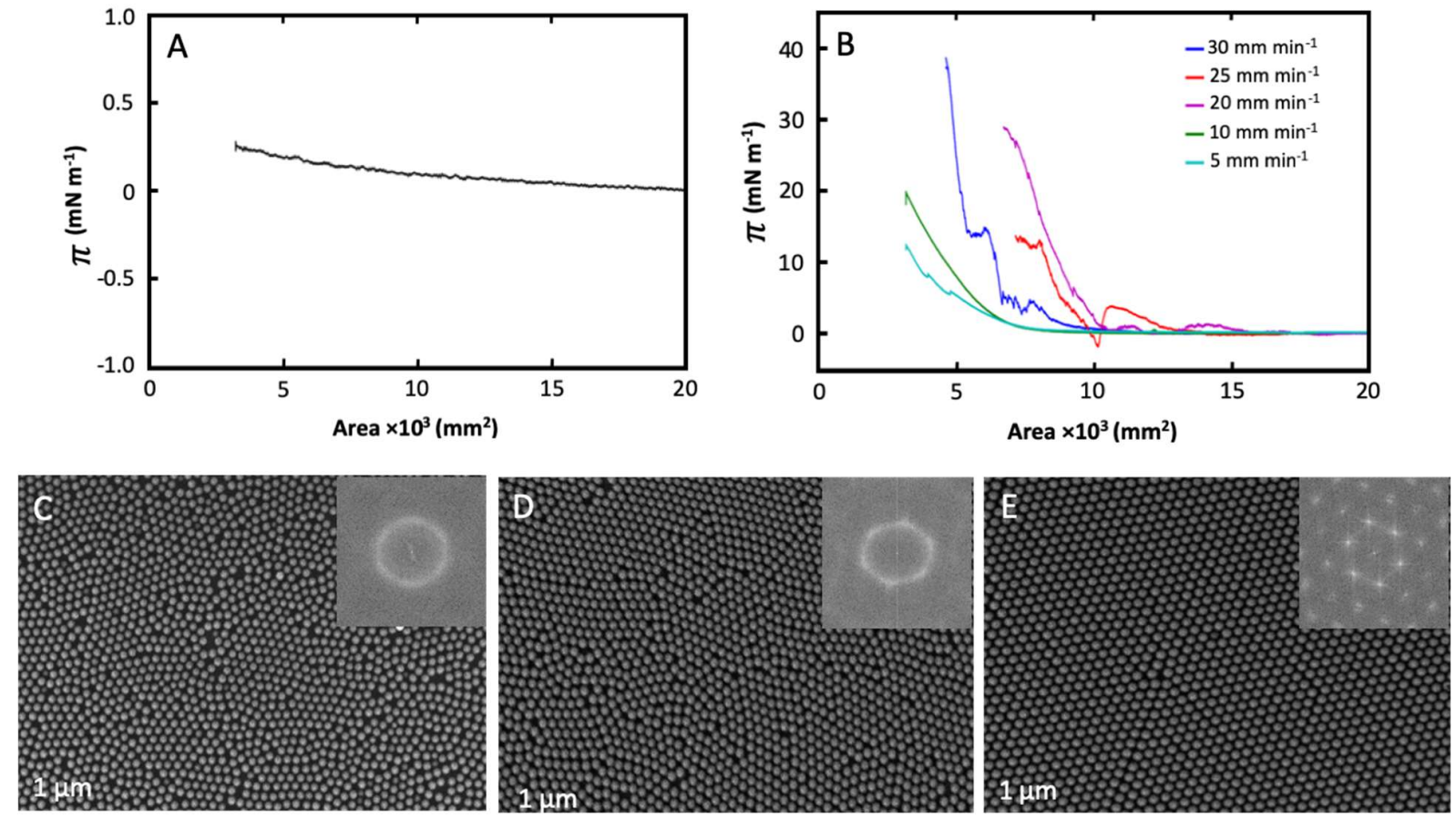

Fig. 7. Optimization of barrier compression and beads transfer speeds: (A) $\pi$-A curve after PS monolayer compression at different speeds from $30 \mathrm{~mm} \mathrm{~min}{ }^{-1}$ to $5 \mathrm{~mm} \mathrm{~min}^{-1}$. SEM and corresponding FFT (insets) images of the monolayer transfer to the substrate at a rate of (B) 20 $\mathrm{mm} \mathrm{min}^{-1}$, (C) $10 \mathrm{~mm} \mathrm{~min}^{-1}$ and (D) $5 \mathrm{~mm} \mathrm{~min}^{-1}$.
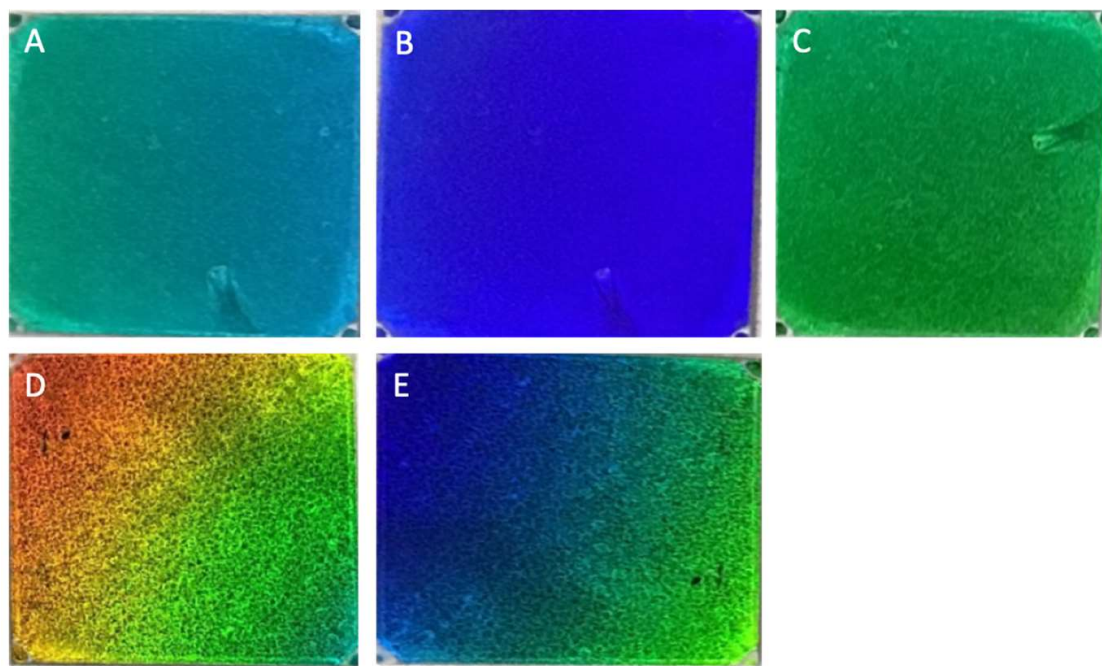

Fig. 8. Digital photographic images or diffraction patterns of the polystyrene colloidal mask after substrate transfer using A-C) $340 \mathrm{~nm}$ and D, E) $1 \mu \mathrm{m}$ beads, observed at different angles of view.

The diffraction colors can be easily observed with naked eye for such a large domain even after transfer onto the substrate, $2.5 \mathrm{~cm}^{2}$ (Fig. 8). Furthermore, the high regularity of the bead 
arrangement was validated with close-up SEM and FFT images recorded at six different positions, including upper third of the mask layer as shown in Fig. 9.
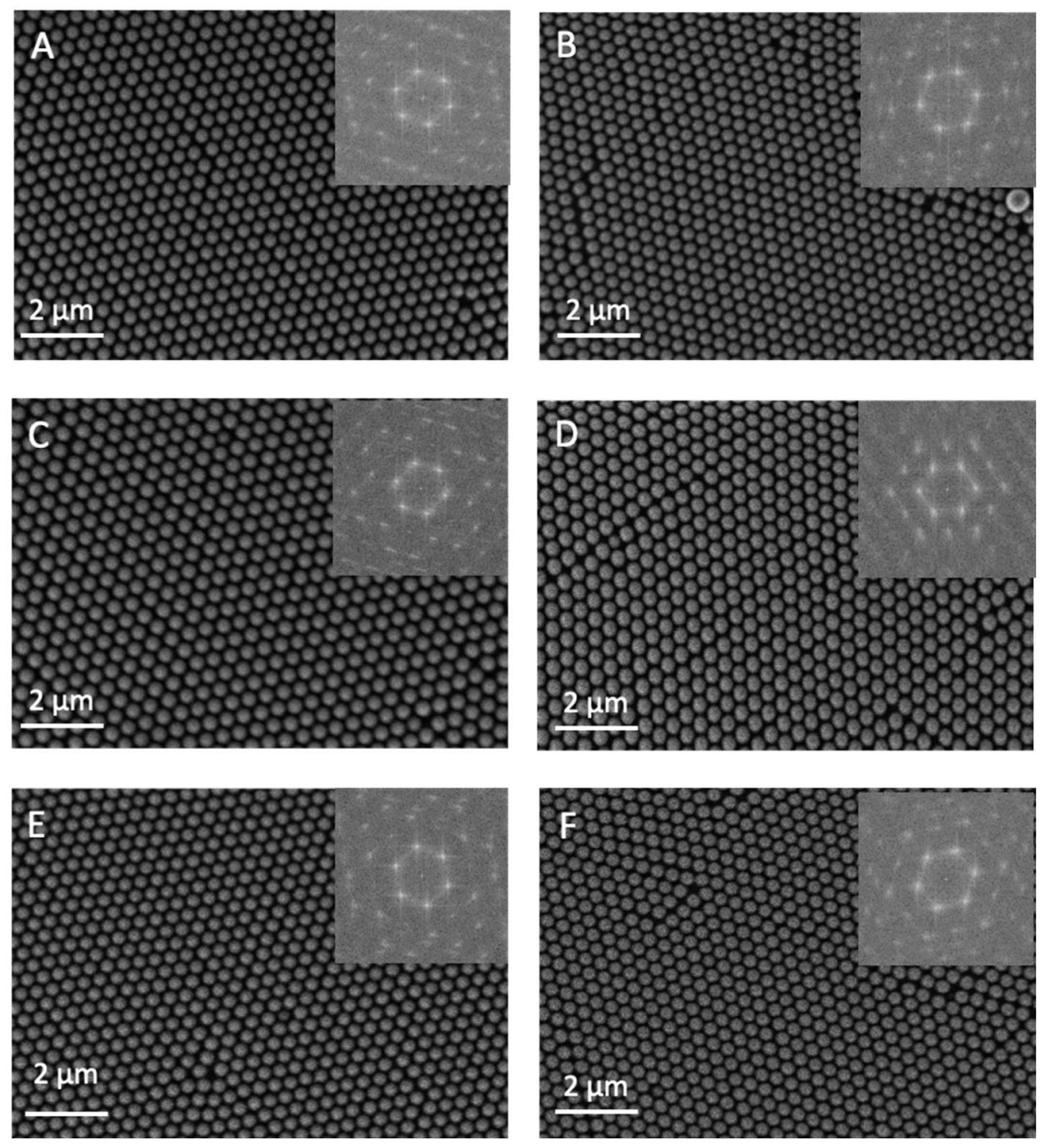

Fig. 9. SEM and FFT images of the PS 2D colloidal mask measured at A-D) the upper third of the mask layer at the corners of the sample, and E, F) two random middle positions on the samples.

After attaining hep monolayer by compression, the glass substrate was submerged into the water using the dip coater at higher speed, $50 \mathrm{~mm} \mathrm{~min}^{-1}$. In a control experiment, the substrate was submerged into the water before compression to minimize disturbances of the compact compressed LB film. However, no significant changes were observed on the quality of the mask transferred to the substrate and therefore, the substrate could also be submerged prior to compression. After 10 min, the substrate was pulled out at different speeds from $5 \mathrm{~mm} \mathrm{~min}^{-1}$ to $20 \mathrm{~mm} \mathrm{~min}^{-1}$. It is worth noting that while transferring the beads to the substrate, the air-water interface was also compressed at the same rate to preserve the hcp arrangement on the substrate as similar as it was 
in the air-water interface. Fig. 7C-E show the SEM images of the mask transferred to the substrate at these different speeds. It is clearly seen from Fig. 7C that at higher transfer speed, the LB film destabilized. At slower speed, the mask seems very well arranged, Fig. 7E. From the FFT image in Fig. 7E (inset), it is evident that the mask transferred to the substrate is a single domain where the lattice direction and the hexagonal order are preserved over the whole area. Furthermore, the excellent honeycomb lattice observed in the Voronoi-diagram (Fig. 10) also confirms the improved hexagonal arrangement of the beads for slower transfer. The present recipe works well for glass/fused silica substrates, however, it can also be used for silicon and polymer surfaces after oxygen plasma and UV treatments respectively or with little modification in the recipe.

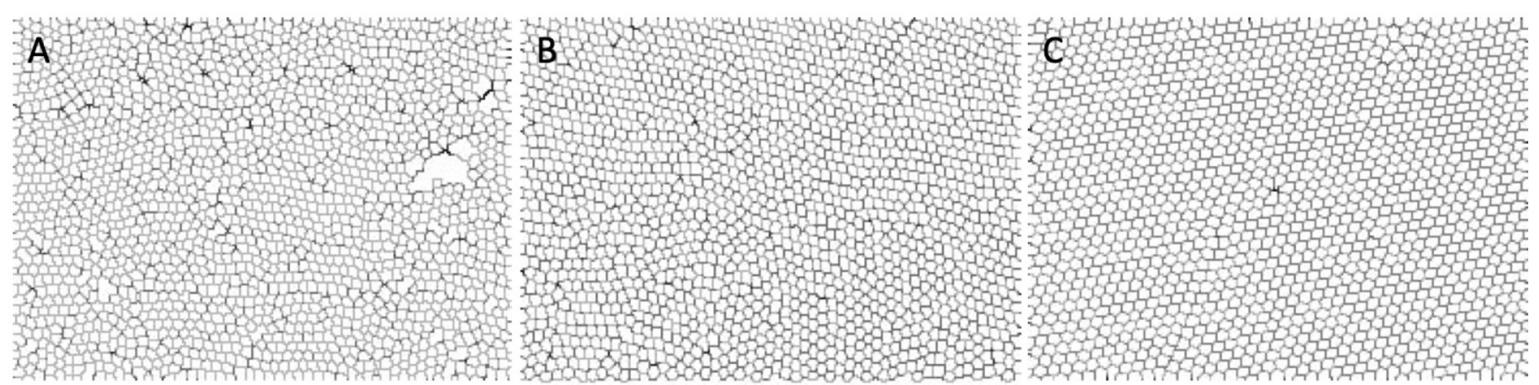

Fig. 10. Voronoi-diagram of the SEM images observed for the beads transferred to the substrate at the speed of A) $20 \mathrm{~mm} \mathrm{~min}^{-1}$, B) $10 \mathrm{~mm} \mathrm{~min}^{-1}$, and C) $5 \mathrm{~mm} \mathrm{~min}$.

\subsection{Aluminium nanostructure realization}

Recently, aluminium has become a highly appealing material to replace conventional plasmonic metals such as gold and silver for photovoltaic and photocatalytic applications due to its abundant availability in earth's crust and lower prize $[59,60]$. The fabrication of aluminium nanostructures over large area using state of the art techniques is extremely challenging and, hence, we desired to use LB technique assisted colloidal lithography for realizing aluminium nanotriangles (AINTs). Homogenously distributed AINTs were obtained by depositing a $40 \mathrm{~nm}$ aluminium thin film, using evaporation, on the well prepared PS colloidal mask followed by a lift-off process. Thereafter, the quality of the nanostructures arrangement was studied using SEM. It is apparent that very well ordered equilateral triangles were obtained as shown in Fig. 11A. Further statistical analysis of the SEM image shows that the average edge length of the AlNTs is about $85 \mathrm{~nm}$ (Fig. 11B) with a surface coverage of $7.2 \%$. The optical property of the AlNTs measured using UV-Vis-NIR spectrometer shows a broad resonance peak at $480 \mathrm{~nm}$ (Fig. 11C) suggesting that it could be tuned 
for harvesting solar energy ranging from near UV to visible light. The spectral position observed for AlNTs agrees well with earlier reports $[2,61]$.
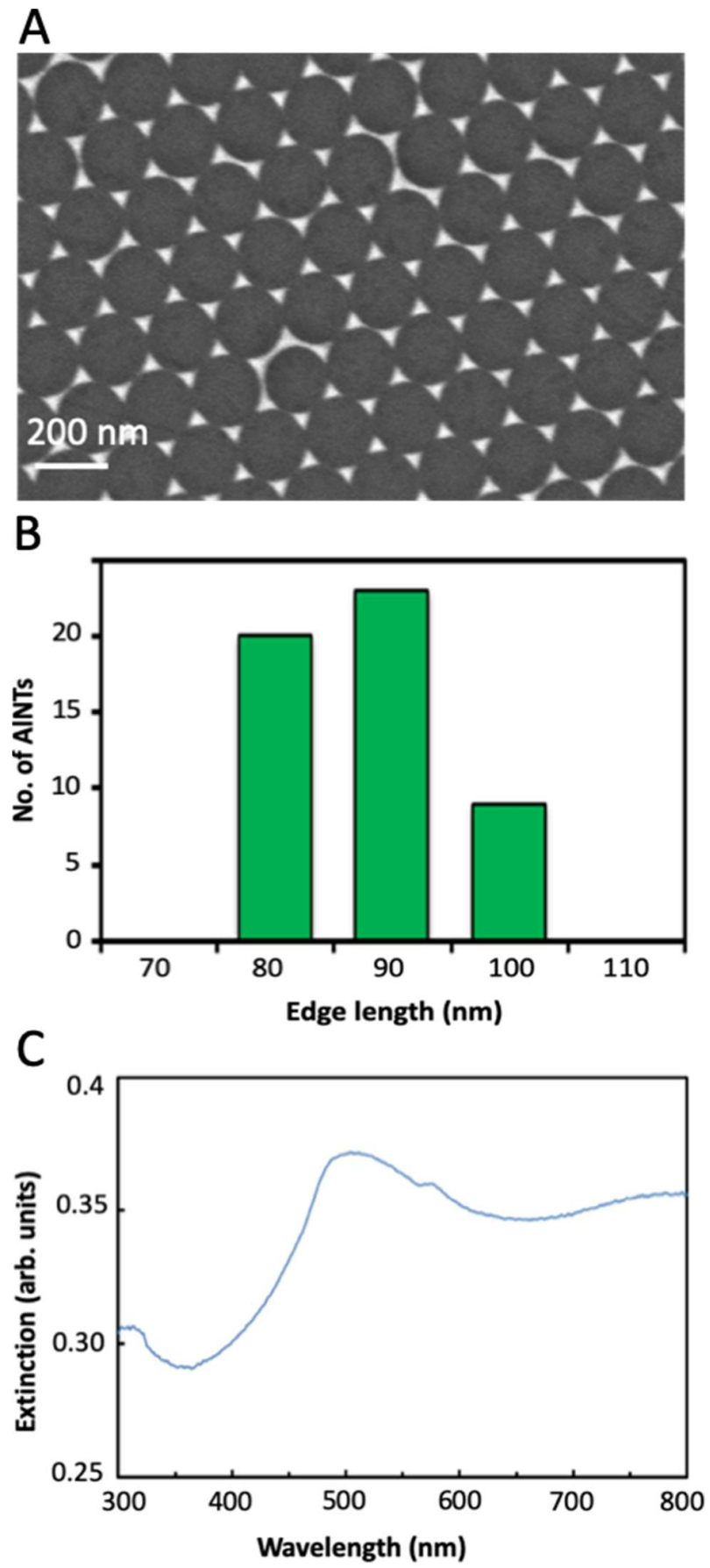

Fig. 11. (A) SEM image of the aluminium nanostructures obtained after lift-off of the mask. (B) AlNTs size distribution. (C) Optical extinction spectra of the AlNTs.

It is worth noting that a 2-3 nm oxide $\left(\mathrm{Al}_{2} \mathrm{O}_{3}\right)$ layer formed on the surface of the $\mathrm{Al}$ does not alter the optical property and limits further oxidation of the metal, thus providing better stability as 
shown in earlier report [2,62]. The present AlNTs can be used solely for plasmonic hot electron generation, and combined with semiconducting material to enhance the charge carrier generation through plasmonic near-field coupling. Various dimension for the Al nanostructures can be obtained by preparing the PS colloidal masks with different beads sizes - using the demonstrated recipe - to tune the plasmonic resonance position for various applications. In addition, different shapes of the metal nanostructures can be fabricated by preparing double layer masks, changing the mask morphology, and evaporation condition.

\section{Conclusion}

In summary, we have studied the usually un-documented methodological subtleties required for the production of a long-range hep PS monolayer at the air-water interface using a Langmuir Blodgett trough. The different parameters involved in LB mask preparation were carefully examined and optimized and we can draw the following recommendations. The commercial PS suspension must be washed using ethanol to improve the hydrophobicity of the particles. No electrolyte is needed in the water subphase for washed beads. The beads suspension must be dispensed using a glass slide angled at $45^{\circ}$. The spreading agent should be mixed with water at 1:1 ratio. A compression speed of $10 \mathrm{~mm} \mathrm{~min}^{-1}$ and mask transfer speed of $5 \mathrm{~mm} \mathrm{~min}^{-1}$ guarantee long-range order. By following the recipe shown here, the reader should easily fabricate latticelike colloidal masks for producing nanostructures over larger areas. The demonstrated method works very nicely for other commercial PS beads with no functionalized group or containing little sulfate anionic charges with better size distribution. 


\section{Acknowledgment}

The authors acknowledge the financial support from the Gebert Ruf Stiftung, Switzerland (Grant no. GRS-039/16) and from the European Research Council (Grant No. ERC-2015-AdG-695206 Nanofactory). 


\section{References}

[1] J. Butet, P.-F. Brevet, O.J.F. Martin, Optical Second Harmonic Generation in Plasmonic Nanostructures: From Fundamental Principles to Advanced Applications, ACS Nano. 9 (2015) 10545-10562.

[2] M. Thangamuthu, C. Santschi, O.J.F. Martin, Photocatalytic ammonia production enhanced by a plasmonic near-field and hot electrons originating from aluminium nanostructures, Faraday Discuss. 214 (2019) 399-415.

[3] S. Dutta Gupta, Plasmonic nanostructures for physical and biological applications, EPF, Lausanne, 2015.

[4] W. Ye, R. Long, H. Huang, Y. Xiong, Plasmonic nanostructures in solar energy conversion, J. Mater. Chem. C. 5 (2017) 1008-1021.

[5] Y. Ekinci, A. Christ, M. Agio, O.J.F. Martin, H.H. Solak, J.F. Löffler, Electric and magnetic resonances in arrays of coupled gold nanoparticle in-tandem pairs, Opt. Express. 16 (2008) $13287-13295$.

[6] Y. Chen, Nanofabrication by electron beam lithography and its applications: A review, Microelectron. Eng. 135 (2015) 57-72.

[7] T. Siegfried, Y. Ekinci, O.J.F. Martin, H. Sigg, Gap Plasmons and Near-Field Enhancement in Closely Packed Sub-10 nm Gap Resonators, Nano Lett. 13 (2013) 5449-5453.

[8] F. Watt, A.A. Bettiol, J.A. Van Kan, E.J. Teo, M.B.H. Breese, Ion beam lithography and nanofabrication: A review, Int. J. Nanosci. 04 (2005) 269-286.

[9] J.T.M. Stevenson, A.M. Gundlach, The application of photolithography to the fabrication of microcircuits, J. Phys. E. 19 (1986) 654-667.

[10] U.C. Fischer, H.P. Zingsheim, Submicroscopic pattern replication with visible light, J. Vac. Sci. Technol. 19 (1981) 881-885.

[11] H.W. Deckman, B. Abeles, J.H. Dunsmuir, C.B. Roxlo, Microfabrication of molecular scale microstructures, Appl. Phys. Lett. 50 (1987) 504-506.

[12] C. Haginoya, M. Ishibashi, K. Koike, Nanostructure array fabrication with a sizecontrollable natural lithography, Appl. Phys. Lett. 71 (1997) 2934-2936.

[13] C.L. Haynes, R.P. Van Duyne, Nanosphere Lithography: A Versatile Nanofabrication Tool for Studies of Size-Dependent Nanoparticle Optics, J. Phys. Chem. B. 105 (2001) 55995611. 
[14] X. Zhang, A. V. Whitney, J. Zhao, E.M. Hicks, R.P. Van Duyne, Advances in Contemporary Nanosphere Lithographic Techniques, J. Nanosci. Nanotechnol. 6 (2006) 1920-1934.

[15] X. Liang, R. Dong, J.C. Ho, Self-Assembly of Colloidal Spheres toward Fabrication of Hierarchical and Periodic Nanostructures for Technological Applications, Adv. Mater. Technol. 4 (2019) 1-19.

[16] V. Lotito, T. Zambelli, Approaches to self-assembly of colloidal monolayers: A guide for nanotechnologists, Adv. Colloid Interface Sci. 246 (2017) 217-274.

[17] R. van Dommelen, P. Fanzio, L. Sasso, Surface self-assembly of colloidal crystals for micro- and nano-patterning, Adv. Colloid Interface Sci. 251 (2018) 97-114.

[18] T.R. Jensen, G.C. Schatz, R.P. Van Duyne, Nanosphere Lithography: Surface Plasmon Resonance Spectrum of a Periodic Array of Silver Nanoparticles by Ultraviolet-Visible Extinction Spectroscopy and Electrodynamic Modeling, J. Phys. Chem. B. 103 (2002) 2394-2401.

[19] Z. Wang, B. Ai, H. Möhwald, G. Zhang, Colloidal Lithography Meets Plasmonic Nanochemistry, Adv. Opt. Mater. 6 (2018) 1800402.

[20] T.R. Jensen, M.D. Malinsky, C.L. Haynes, R.P. Van Duyne, Nanosphere Lithography: Tunable Localized Surface Plasmon Resonance Spectra of Silver Nanoparticles, J. Phys. Chem. B. 104 (2000) 10549-10556.

[21] G. Klös, A. Andersen, M. Miola, H. Birkedal, D.S. Sutherland, Oxidation controlled lift-off of 3D chiral plasmonic Au nano-hooks, Nano Res. 12 (2019) 1635-1642.

[22] V.E. Bochenkov, D.S. Sutherland, Chiral plasmonic nanocrescents: large-area fabrication and optical properties, Opt. Express. 26 (2018) 27101.

[23] S. Cataldo, J. Zhao, F. Neubrech, B. Frank, C. Zhang, P. V. Braun, H. Giessen, Hole-mask colloidal nanolithography for large-area low-cost metamaterials and antenna-assisted surface-enhanced infrared absorption substrates, ACS Nano. 6 (2012) 979-985.

[24] J. Zhao, B. Frank, S. Burger, H. Giessen, Large-area high-quality plasmonic oligomers fabricated by angle-controlled colloidal nanolithography, ACS Nano. 5 (2011) 9009-9016.

[25] H. Fredriksson, Y. Alaverdyan, A. Dmitriev, C. Langhammer, D.S. Sutherland, M. Zäch, B. Kasemo, Hole-mask colloidal lithography, Adv. Mater. 19 (2007) 4297-4302.

[26] K. Chen, T. Duy Dao, T. Nagao, Tunable Nanoantennas for Surface Enhanced Infrared 
Absorption Spectroscopy by Colloidal Lithography and Post-Fabrication Etching, Sci. Rep. 7 (2017) 44069.

[27] A. Kosiorek, W. Kandulski, H. Glaczynska, M. Giersig, Fabrication of Nanoscale Rings, Dots, and Rods by Combining Shadow Nanosphere Lithography and Annealed Polystyrene Nanosphere Masks, Small. 1 (2005) 439-444.

[28] Y. Lu, G.L. Liu, J. Kim, Y.X. Mejia, L.P. Lee, Nanophotonic Crescent Moon Structures with Sharp Edge for Ultrasensitive Biomolecular Detection by Local Electromagnetic Field Enhancement Effect, Nano Lett. 5 (2005) 119-124.

[29] N. Vogel, M. Jung, N.L. Bocchio, M. Retsch, M. Kreiter, I. Köper, Reusable localized surface plasmon sensors based on ultrastable nanostructures, Small. 6 (2010) 104-109.

[30] X. Xu, Q. Yang, N. Wattanatorn, C. Zhao, N. Chiang, S.J. Jonas, P.S. Weiss, MultiplePatterning Nanosphere Lithography for Fabricating Periodic Three-Dimensional Hierarchical Nanostructures, ACS Nano. 11 (2017) 10384-10391.

[31] N. Vogel, S. Goerres, K. Landfester, C.K. Weiss, A Convenient Method to Produce Closeand Non-close-Packed Monolayers using Direct Assembly at the Air-Water Interface and Subsequent Plasma-Induced Size Reduction, Macromol. Chem. Phys. 212 (2011) 17191734.

[32] S.S. Shinde, S. Park, Oriented colloidal-crystal thin films of polystyrene spheres via spin coating, J. Semicond. 36 (2015) 23001

[33] C. Zhang, S. Cvetanovic, J.M. Pearce, Fabricating ordered 2-D nano-structured arrays using nanosphere lithography, MethodsX. 4 (2017) 229-242.

[34] Z. Lu, M. Zhou, Fabrication of large scale two-dimensional colloidal crystal of polystyrene particles by an interfacial self-ordering process, J. Colloid Interface Sci. 361 (2011) 429 435.

[35] W. Ruan, Z. Lu, N. Ji, C. Wang, B. Zhao, J. Zhang, Facile Fabrication of Large Area Polystyrene Colloidal Crystal Monolayer via Surfactant-free Langmuir-Blodgett Technique, Chem. Res. Chinese Univ. 23 (2007) 712-714.

[36] M. Bardosova, M.E. Pemble, I.M. Povey, R.H. Tredgold, The Langmuir-Blodgett Approach to Making Colloidal Photonic Crystals from Silica Spheres, Adv. Mater. 22 (2010) 3104 3124.

[37] K.-U. Fulda, B. Tieke, Monolayers of mono- and bidisperse spherical polymer particles at 
the air/water interface and Langmuir-Blodgett layers on solid substrates, Supramol. Sci. 4 (1997) 265-273.

[38] A. Agod, N. Nagy, Z. Hórvölgyi, Modeling the Structure Formation of Particulate Langmuir Films: the Effect of Polydispersity, Langmuir. 23 (2007) 5445-5451.

[39] M. Bardosova, F.C. Dillon, M.E. Pemble, I.M. Povey, R.H. Tredgold, Langmuir-Blodgett assembly of colloidal photonic crystals using silica particles prepared without the use of surfactant molecules, J. Colloid Interface Sci. 333 (2009) 816-819.

[40] N. Nagy, A.E. Pap, E. Horváth, J. Volk, I. Bársony, A. Deák, Z. Hórvölgyi, Large area selfassembled masking for photonic applications, Appl. Phys. Lett. 89 (2006) 63104.

[41] S. Reculusa, P. Massé, S. Ravaine, Three-dimensional colloidal crystals with a well-defined architecture, J. Colloid Interface Sci. 279 (2004) 471-478.

[42] B. van Duffel, R.H.A. Ras, F.C. De Schryver, R.A. Schoonheydt, Langmuir-Blodgett deposition and optical diffraction of two-dimensional opal, J. Mater. Chem. 11 (2001) $3333-3336$.

[43] D.F. Williams, J.C. Berg, The aggregation of colloidal particles at the air-water interface, J. Colloid Interface Sci. 152 (1992) 218-229.

[44] D.J. Robinson, J.C. Earnshaw, Initiation of aggregation in colloidal particle monolayers, Langmuir. 9 (1993) 1436-1438.

[45] G. Tolnai, A. Agod, M. Kabai-Faix, A.L. Kovács, J.J. Ramsden, Z. Hórvölgyi, Evidence for Secondary Minimum Flocculation of Stöber Silica Nanoparticles at the Air-Water Interface: Film Balance Investigations and Computer Simulations, J. Phys. Chem. B. 107 (2003) 11109-11116.

[46] I. Dékány, J. Németh, M. Szekeres, R. Schoonheydt, Surfacial, liquid sorption and monolayer-forming properties of hydrophilic and hydrophobic Stöber silica particles, Colloid Polym. Sci. 282 (2003) 1-6.

[47] D. Truzzolillo, H. Sharaf, U. Jonas, B. Loppinet, D. Vlassopoulos, Tuning the Structure and Rheology of Polystyrene Particles at the Air-Water Interface by Varying the $\mathrm{pH}$, Langmuir. 32 (2016) 6956-6966.

[48] C.E. McNamee, S. Yamamoto, H.-J. Butt, K. Higashitani, A Straightforward Way To Form Close-Packed TiO2 Particle Monolayers at an Air/Water Interface, Langmuir. 27 (2011) 887-894. 
[49] H.-L. Nie, X. Dou, Z. Tang, H.D. Jang, J. Huang, High-Yield Spreading of Water-Miscible Solvents on Water for Langmuir-Blodgett Assembly, J. Am. Chem. Soc. 137 (2015) 10683-10688.

[50] S. Reculusa, S. Ravaine, Synthesis of Colloidal Crystals of Controllable Thickness through the Langmuir-Blodgett Technique, Chem. Mater. 15 (2003) 598-605.

[51] P. V Petkov, K.D. Danov, P.A. Kralchevsky, Surface Pressure Isotherm for a Monolayer of Charged Colloidal Particles at a Water/Nonpolar-Fluid Interface: Experiment and Theoretical Model, Langmuir. 30 (2014) 2768-2778.

[52] N. Vogel, L. de Viguerie, U. Jonas, C.K. Weiss, K. Landfester, Wafer-Scale Fabrication of Ordered Binary Colloidal Monolayers with Adjustable Stoichiometries, Adv. Funct. Mater. 21 (2011) 3064-3073.

[53] F. Ghezzi, J.C. Earnshaw, M. Finnis, M. McCluney, Pattern Formation in Colloidal Monolayers at the Air-Water Interface, J. Colloid Interface Sci. 238 (2001) 433-446.

[54] K.J.M. Bishop, C.E. Wilmer, S. Soh, B.A. Grzybowski, Nanoscale Forces and Their Uses in Self-Assembly, Small. 5 (2009) 1600-1630.

[55] S. Fujii, M. Kappl, H.-J. Butt, T. Sugimoto, Y. Nakamura, Soft Janus Colloidal Crystal Film, Angew. Chemie Int. Ed. 51 (2012) 9809-9813.

[56] C.-C. Ho, P.-Y. Chen, K.-H. Lin, W.-T. Juan, W.-L. Lee, Fabrication of Monolayer of Polymer/Nanospheres Hybrid at a Water-Air Interface, ACS Appl. Mater. Interfaces. 3 (2011) 204-208.

[57] C.A. Schneider, W.S. Rasband, K.W. Eliceiri, NIH Image to ImageJ: 25 years of image analysis, Nat. Methods. 9 (2012) 671-675.

[58] H. Ko, H.-W. Lee, J. Moon, Fabrication of colloidal self-assembled monolayer (SAM) using monodisperse silica and its use as a lithographic mask, Thin Solid Films. 447-448 (2004) $638-644$.

[59] L. Zhou, C. Zhang, M.J. McClain, A. Manjavacas, C.M. Krauter, S. Tian, F. Berg, H.O. Everitt, E.A. Carter, P. Nordlander, N.J. Halas, Aluminum Nanocrystals as a Plasmonic Photocatalyst for Hydrogen Dissociation, Nano Lett. 16 (2016) 1478-1484.

[60] P.K. Parashar, R.P. Sharma, V.K. Komarala, Plasmonic silicon solar cell comprised of aluminum nanoparticles: Effect of nanoparticles' self-limiting native oxide shell on optical and electrical properties, J. Appl. Phys. 120 (2016) 143104. 
[61] D. Gérard, S.K. Gray, Aluminium plasmonics, J. Phys. D. Appl. Phys. 48 (2014) 184001.

[62] K. Thyagarajan, C. Santschi, P. Langlet, O.J.F. Martin, Highly Improved Fabrication of Ag and Al Nanostructures for UV and Nonlinear Plasmonics, Adv. Opt. Mater. 4 (2016) 871876. 